\title{
Model Experiment on the Coagulation of Inclusion Particles in Liquid Steel
}

\author{
Shoji TANIGUCHI, Atsushi KIKUCHI, Tomoko (SE $^{1)}$ and Naruhito SHOJI ${ }^{2)}$
}

Faculty of Engineering, Tohoku University, Aza Aoba, Aramaki, Aoba-ku, Sendai, Miyagi-ken, 980-77 Japan. 1) Formerly Graduate School, Tohoku University. Now at Sanyo Special Steel Co., Ltd., Nakashima, Shikama-ku, Himeji, Hyogo-ken, 672 Japan. $\quad$ 2) Formerly Graduate School, Tohoku University. Now at Sumitomo Metal Industries, Ltd., Sunayama, Hasaki-machi, Kashima, Ibaraki-ken, 314-02 Japan.

(Received on May 15, 1996; accepted in final form on September 9, 1996)

\begin{abstract}
Model experiments on the particle coagulation in $\mathrm{NaCl}$-aqueous solutions were made in an agitated vessel to learn the coagulation rate of inclusion particles in liquid steel. Polystyrene-latex particles, silica particles and alumina particles were tested at various agitation speeds, and coagulation coefficients were obtained by comparing the observed coagulation rate with the predicted rate by the Saffman-Turner model on turbulent collision. The Higashitani theory which considered the effect of van der Waals force and viscous resistance force on the coagulation coefficient was applied to obtain the Hamaker constant from the measured coefficients. Based on the measured Hamaker constants, the coagulation coefficients of inclusion particles in liquid steel were predicted for several steelmaking processes with different stirring intensities.
\end{abstract}

KEY WORDS: turbulent coagulation; inclusion; clean steel; deoxidation; energy dissipation rate; stirring power density; Hamaker constant.

\section{Introduction}

Inclusion particles in liquid steel are known to be coagulated by turbulent collision, and as a result enlarged particles become easy to be removed from liquid steel. Therefore, it is quite important to understand the phenomenon of turbulent coagulation in detail. The purpose of the present study was to elucidate the mechanism of inclusion coagulation in liquid steel by applying the theory of turbulent collision which was developed in the field of colloid engineering.

Many studies have been made of the coagulation and elimination of inclusion particles in refining vessels ${ }^{1-4)}$ and tundishes for continuous casting. ${ }^{5,6)}$ The SaffmanTurner model ${ }^{7)}$ was applied in these studies to predict the turbulent coagulation rate. However, Nakanishi and Szekely ${ }^{3)}$ pointed out that a correction factor, $0.27-0.63$, should be introduced to the Saffman-Turner model for estimating deoxidation rates. In the colloid-engineering field, Higashitani et al. ${ }^{8)}$ investigated the effects of viscous force and van der Waals force on the turbulent coagulation of polystyrene latex in aqueous solution. They proposed a correlation between the correction factor of the Saffman-Turner model and the above two forces.

In this study, the applicability of the Higashitani model was verified first, and then the role of the van der Waals force on the coagulation of silica and alumina was discussed experimentally.

\section{Theory of Turbulent Collision and Interaction between} Particles

Collision frequency between a pair of particles $i$ and $j$ in a unit volume is given by

$$
N_{i j}=1.3\left(a_{i}+a_{j}\right)^{3}(\varepsilon / v)^{1 / 2} n_{i} n_{j}
$$

where $a$ is the particle radus, $\varepsilon$ energy-dissipation rate, $v$ kinematic viscosity, and $n$ particle-number density. It is important to note that the above equation is valid when the collision radius, $a_{i}+a_{j}$, is far less than the Kolmogoroff's microscale, $\eta$.

$$
a_{i}+a_{j} \ll \eta ; \quad \eta \equiv\left(v^{3} / \varepsilon\right)^{1 / 4}
$$

The change in size and concentration of coagulated particles with time can be expressed by the following population-balance equation, ${ }^{9)}$

$$
\frac{d n_{k}}{d t}=\frac{1}{2} \sum_{i=1, i+j=k}^{i=k-1} N_{i j}-\sum_{i=1}^{\infty} N_{i k}
$$

where $n_{k}$ is the concentration of the coagulated particle composed of $k$-pieces of primary particle. The terms on the right-hand side of Eq. (3) are the generation rate and disappearance rate of $k$-particle, respectively.

Higashitani et al. ${ }^{8)}$ introduced the coagulation coefficient, $\alpha$, to the Saffman-Turner model as follows:

$$
N_{i j}=\alpha\left[1.3\left(a_{i}+a_{j}\right)^{3}(\varepsilon / v)^{1 / 2} n_{i} n_{j}\right]
$$

Under the rapid coagulation condition, they correlated $\alpha$ with a dimensionless parameter, $N_{\mathrm{T}}$, which represents 
the ratio between the viscous force and the van der Waals force,

$$
\alpha=f\left(N_{\mathrm{T}}\right) ; \quad N_{\mathrm{T}}=\frac{6 \pi \mu a_{1}^{3}(4 \varepsilon / 15 \pi v)^{1 / 2}}{A}
$$

where $A$ is the Hamaker constant which is the measure of the van der Waals force between particles described by the following equation, ${ }^{10}$ )

$$
A=\pi^{2} q_{1}^{2} \beta_{11}
$$

Here, $q_{1}$ is the number of molecules in a unit volume and $\beta_{11}$ is the constant in London's equation for the interaction between two atoms. Hamaker constants in the literature are so widely scattered that we decided to measure them using an agitated vessel.

\section{Experiment}

\subsection{Experimental Method}

The agitated vessel used was composed of a cylindrical glass vessel $(750 \mathrm{ml})$, a two-blade impeller $(42 \mathrm{~mm}$ diameter) and four baffle plates. In the experiment, particles suspended in distilled water were put into an electrolyte solution agitated in the vessel. At suitable time intervals, a small amount of the solution was taken, and the particle-number density was measured by the electrical sensing zone method (Coulter counter). Three kinds of particles were used in the experiment, polystyrene latex (PSL), alumina and silica. Although PSL and silica particles are spherical, the shape of alumina particles was irregular. Particle diameters were: PSL: $2.0 \mu \mathrm{m}$, silica: $2.8 \mu \mathrm{m}$, and alumina: $1.8 \mu \mathrm{m}$. A rapid coagulation condition was obtained using $\mathrm{NaCl}$-aqueous solution $\left(2-3 \mathrm{kmol} / \mathrm{m}^{3}\right)$ having proper $\mathrm{pH}$ (PSL: $7, \mathrm{SiO}_{2}$ : $\left.3, \mathrm{Al}_{2} \mathrm{O}_{3}: 8-9.4\right)$. The agitation speed was changed from 400 to 1000 r.p.m.

Rushton's graph ${ }^{11)}$ was applied to the estimation of $\varepsilon$. From the definition of the power number, $N_{\mathrm{P}}$, the following equation is given,

$$
\varepsilon_{0}=N_{\mathrm{P}} \rho n^{3} d_{\mathrm{s}}^{5} / M
$$

where $n$ is agitation speed, $\rho$ liquid density, $d_{\mathrm{s}}$ diameter of impeller, and $M$ mass of liquid. Considering the fact that not all of the energy input dissipates in the liquid contained in the agitated vessel, the following equation was adopted in the present study, which was recommended by Higashitani et al.

$$
\varepsilon=0.15 \varepsilon_{0}
$$

\subsection{Results and Discussion}

Figure 1 shows coagulation curves of PSL obtained for various agitation speeds. The vertical axis gives the total number density of particles in liquid normalized by the initial number density. The value of $N_{\mathrm{t}} / N_{0}$ decreases monotonously and approaches zero as the time increases, which means that the breakup of the agglomerates is negligible. Coagulation curves in Fig. 1 are compared with the calculated curve in Fig. 2. The method of calculating coagulation curves was the same as used in the previous study, ${ }^{8)}$ that is, the population balance equation, Eq. (3), was solved by the Runge-Kutta-Gill

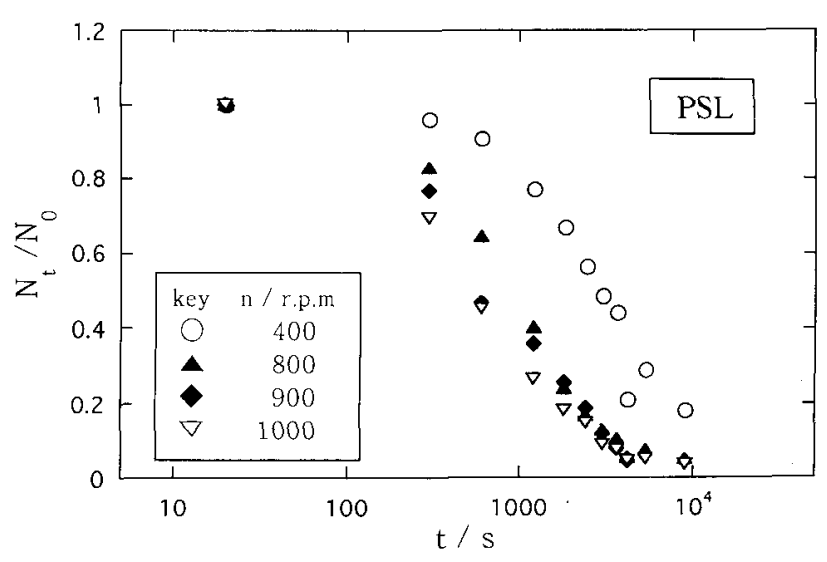

Fig. 1. Coagulation curves of PSL at different agitation speeds.

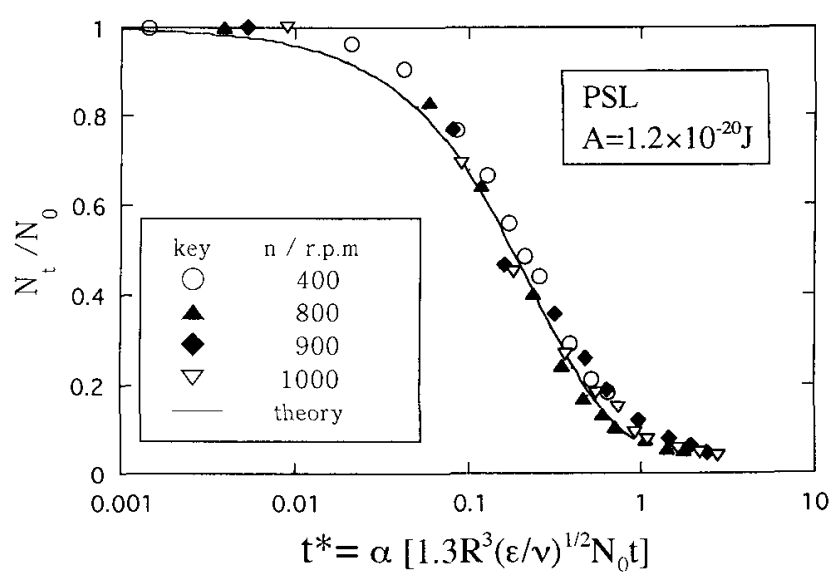

Fig. 2. Normalized coagulation curves of PSL obtained at different agitation speeds.

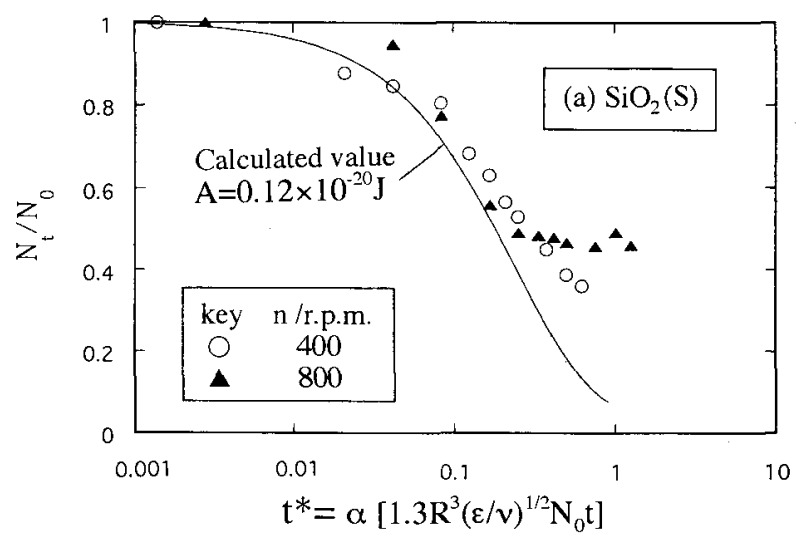

Fig. 3. Normalized coagulation curves of $\mathrm{SiO}_{2}$ obtained at different agitation speeds.

method by considering agglomerates composed of 200 pieces of primary particle. The time scale was normalized using $R\left(=a_{\mathrm{i}}+a_{j}\right), N_{0}, \varepsilon$, and $\alpha$. By selecting a suitable value for the Hamaker constant, $A$, in Eq. (5), all the observed curves fit the calculated curve. The Hamaker constant for PSL could then be determined as $1.2 \times 10^{-20} \mathrm{~J}$ which seems reasonable in comparison with the previous value, $(0.1-9) \times 10^{-20} \mathrm{~J}^{10}$ )

By the same procedure, the Hamaker constants of silica and alumina were determined as shown in Figs. 3 and 4 . In the case of silica at higher agitation speed, the particle number did not decrease below a certain value, viz 0.4 ; this can be attributed to the breakup of ag- 


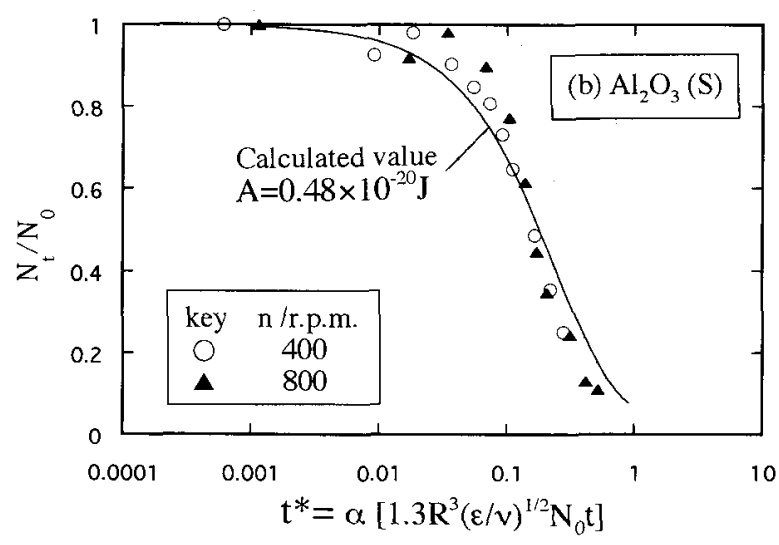

Fig. 4. Normalized coagulation curves of $\mathrm{Al}_{2} \mathrm{O}_{3}$ obtained at different agitation speeds.

Table 1. Hamaker constants obtained in this study.

\begin{tabular}{cccc}
\hline & $\mathrm{PSL}$ & $\mathrm{SiO}_{2}$ & $\mathrm{Al}_{2} \mathrm{O}_{3}$ \\
\hline $\begin{array}{c}A_{131} / 10^{-20} \mathrm{~J} \text { in water } \\
(298 \mathrm{~K})\end{array}$ & 1.2 & 0.12 & 0.48 \\
$\begin{array}{c}A_{11} / 10^{-20} \mathrm{~J} \text { in vacuum } \\
(298 \mathrm{~K})\end{array}$ & 9.6 & 5.5 & 7.2 \\
$\begin{array}{c}A_{131} / 10^{-20} \mathrm{~J} * \text { in liquid iron } \\
(1873 \mathrm{~K})\end{array}$ & - & 3.1 & 2.3
\end{tabular}

* $\rho_{1873 \mathrm{~K}} / \rho_{298 \mathrm{~K}}=0.89$ (iron); $1 \quad\left(\mathrm{SiO}_{2}\right) ; 0.96\left(\mathrm{Al}_{2} \mathrm{O}_{3}\right) A_{33}$ at $298 \mathrm{~K}=4.0 \times 10^{-20} \mathrm{~J}$ (water); $21.2 \times 10^{-20} \mathrm{~J}$ (iron).

glomerates. Around this value, coagulation and breakup seem to be balanced. Breakup in alumina was not observed because of the larger Hamaker constant than silica.

Hamaker constant of particle 1 in medium 3 can be expressed by the following equation, ${ }^{10}{ }^{0}$

$$
A_{131}=\left(\sqrt{A_{11}}-\sqrt{A_{33}}\right)^{2}
$$

where $A_{11}$ and $A_{33}$ are the intrinsic value for materials 1 and 3 , respectively. Substituting the Hamaker constant of water, $A_{33}=4.0 \times 10^{-20} \mathrm{~J}$, into the above equation, the intrinsic constants of PSL, silica and alumina were obtained. These values are shown in Table 1 together with the value of $A_{131}$.

\subsection{Hamaker Constant in Liquid Steel}

The Hamaker constants in liquid steel were predicted based on the obtained intrinsic Hamaker constants of silica and alumina. It is known that the van der Waals force has very weak temperature dependence. ${ }^{12)}$ If London's constant, $\beta_{11}$, in Eq. (7) does not change with temperature, the value of $A_{11}$ and $A_{33}$ at high temperature, e.g. $1873 \mathrm{~K}$, can be estimated by Eq. (6) by considering the change in $q_{1}$ with temperature. Using these values and Eq. (9), the values of $A_{131}$ of silica and alumina in liquid steel were predicted. The results are shown in Table 1. It is found that the values of $A_{131}$ in liquid iron are about 10 fold larger than those in water.

Using the predicted values of $A_{131}$ in liquid iron, the relation between $\alpha$ and $\varepsilon$ for liquid steel was derived and is shown in Fig. 5. As the value of $\varepsilon$ increases, the value of $\alpha$ decreases gradually.

In steady state, the input-stirring energy should be

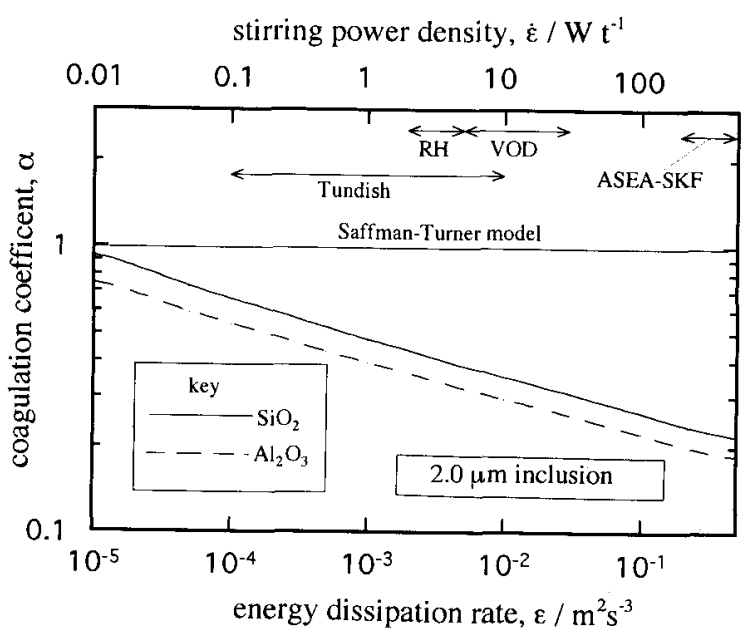

Fig. 5. Estimated values of coagulation coefficient as a function of $\varepsilon$ and $\dot{\varepsilon}$ for $\mathrm{SiO}_{2}$ and $\mathrm{Al}_{2} \mathrm{O}_{3}$ particles in liquid steel.

equivalent to the dissipation energy at the smallest eddies. On the upper abscissa in Fig. 5, the stirring power density, $\varepsilon$, is indicated instead of the energy-dissipation rate. The value of $\varepsilon$ for metallurgical processes evaluated in the previous study ${ }^{13)}$ are shown in the same figure. If this figure is applied, the turbulent coagulation in steelmaking processes seems to be understood in more detail than before.

\section{Conclusions}

The mechanism of the turbulent coagulation of inclusion particles in liquid steel was investigated using the water-model experiment, and the following results were obtained.

(1) By comparing the observed coagulation curves with the calculated curve obtained by the theory of Higashitani et al., the Hamaker consatant of polystyrene latex was determined to be $A_{131}=1.2 \times 10^{-20} \mathrm{~J}$.

(2) The Hamaker constants of silica and alumina particles in water were determined to be $A_{131}\left(\mathrm{SiO}_{2}\right)=$ $0.12 \times 10^{-20} \mathrm{~J}$ and $A_{131}\left(\mathrm{Al}_{2} \mathrm{O}_{3}\right)=0.48 \times 10^{-20} \mathrm{~J}$. In silica particles breakup of agglomerates was observed at high agitation speed.

(3) Hamaker constants in liquid iron were predicted from the presently obtained data by ignoring the temperature dependence of London's coefficient, $\beta_{11}$, included in the Hamaker constant.

\section{Acknowledgments}

The authors are grateful to Messrs. Shinsuke Kano, Toru Sato, Makoto Kobayashi, former undergraduate students in Tohoku Univ., for their assistance, and to Dr. Shinnosuke Usui, former Professor of IAMP, Tohoku University, for helpful comments and discussions. They also wish to thank Dr. Yutaka Matsuyama of Toshiba Ceramics Co., Ltd. for providing spherical silica particles.

\section{REFERENCES}

1) U. Lindborg and K. Torssell: Trans. Metall. Soc. AIME, 242 (1968), 94.

2) S. Linder: Scand. J. Metall., 3 (1974), 137. 
ISIJ International, Vol. 36 (1996), Supplement

3) K. Nakanishi and J. Szekely: Trans. Iron Steel Inst. Jpn., 15 (1975), 522.

4) K. Shirabe and J. Szekely: Trans. Iron Steel Inst. Jpn., 23 (1983), 465.

5) O. J. Ilegbushi and J. Szekely: ISIJ Int., 29 (1989), 1031.

6) A. K. Shinha and Y. Sahai: ISIJ Int., 33 (1993), 556.

7) P. G. Saffman and J. S. Turner: J. Fluid Mech., 1 (1956), 16.

8) K. Higashitani, K. Yamaguchi, Y. Matsuno and G. Hosokawa: J. Chem. Eng. Jpn., 16 (1983), 299.
9) M. Smolchowski: Z. Phys. Chem., 92 (1917), 129, 155.

10) J. Visser: Adv. Colloids Interface Sci., 3 (1972), 331.

11) J. H. Rushton, E. W. Costish and H. J. Everett: Chem. Eng. Prog., 46 (1950), 467.

12) I. W. Osborne-Lee: Particles on Surface 1, ed. by K. L. Mittal, Plenum Press, New York and London, (1988), 77.

13) K. Nakanishi, T. Fujii and J. Szekely: Ironmaking Steelmaking, 2 (1975), 193. 